\title{
REVISÃO SISTEMÁTICA SOBRE O USO DE METODOLOGIAS ATIVAS NOS CURSOS DE ENGENHARIAS NO BRASIL
}

Valéria Cristina Palmeira Zago - valeriazago@cefetmg.br Centro Federal de Educação Tecnológica de Minas Gerais Av. Amazonas, 5253 - Nova Suíça 30421-169 - Belo Horizonte - MG

Elizabeth Regina Halfeld da Costa - elizabethhalfeld@cefetmg.br Centro Federal de Educação Tecnológica de Minas Gerais Av. Amazonas, 5253 - Nova Suíça 30421-169 - Belo Horizonte - MG

Carmem Elisa A. Oliveira Malta - cmms608@gmail.com Centro Federal de Educação Tecnológica de Minas Gerais Av. Amazonas, 5253 - Nova Suíça 30421-169 - Belo Horizonte - MG

Raphael Tobias de Vasconcelos Barros - raphael@desa.ufmg.br Universidade Federal de Minas Gerais Av. Antônio Carlos, 6.627

31270-901 - Belo Horizonte -MG

Resumo: As Diretrizes Curriculares Nacionais do Curso de Graduação em Engenharia explicitam uma clara orientação para a adoção das metodologias ativas. O presente trabalho propôs-se a realizar uma revisão sistemática sobre o uso dessas metodologias nos cursos de engenharia no Brasil, no período de 2011-2020. A busca bibliográfica foi realizada nas bases Web of Science e Scopus, sendo identificados 143 trabalhos publicados em periódicos e eventos científicos. Após a exportação dos trabalhos para o software Start, 74 foram analisados conforme critérios previamente estabelecidos. Grande parte deles (85\%) foi publicada em anais de eventos científicos e $65 \%$ foram realizados por instituições de ensino superior públicas. 53\% são ligados às instituições do Sudeste do país, sendo que o Centro-oeste, Sul e Nordeste apresentaram percentagens de 18, 15 e 13, respectivamente, enquanto que a região Norte, apenas $01 \%$. Observou-se uma predominância do uso da metodologia baseada em projetos e em equipes, seguidamente da metodologia baseada em problemas. Os cursos de engenharias de computação e de software são os que mais tiveram trabalhos publicados (23\%), seguidos das engenharias elétrica e mecânica (17\%), engenharias civil, de produção civil, de produção e ambiental (16\%) e engenharias química e industrial (15\%). Observou-se uma preponderância da utilização em disciplinas profissionalizantes, de forma isolada, em turmas com até 50 alunos e que a grande maioria dos trabalhos apresenta análise da percepção discente sobre o uso da metodologia. Conclui-se que ainda é necessário fomentar o caráter inter e multidisciplinar nos currículos, desenvolvendo habilidades e competências mais complexas, que inclua todo o corpo discente.

Palavras-chave: Ensino-aprendizagem. Diretrizes curriculares. Aprendizagem ativa. Estado da arte. 


\section{INTRODUÇÃO}

A pedagogia dominante na educação em engenharia ainda tem sido aulas expositivas em sala e complementação por meio de resolução de exercícios numéricos e práticas de laboratório, apesar de toda a pesquisa em educação demonstrar a sua ineficácia. Isso conduz os estudantes a adquirirem apenas as habilidades necessárias para conseguirem aprovação em provas e testes (VILLAS-BOAS et al., 2011; RODRIGUES DA SILVA, 2010), além de poder favorecer a evasão escolar.

No entanto, esse cenário já há muito é questionado e, recentemente, explicitamente incentivado a mudar no Brasil, haja vista a vigente normativa sobre as Diretrizes Curriculares Nacionais (DCN) do Curso de Graduação em Engenharia (Res.MEC/CNE/CES no 02/19), que orienta a adoção das metodologias ativas, como forma de promover uma educação mais centrada no aluno. As DCN explanam que o perfil do egresso do curso de graduação em engenharia deve compreender as seguintes características: ter visão holística e humanista, ser crítico, reflexivo, criativo, cooperativo e ético e com forte formação técnica; estar apto a pesquisar, desenvolver, adaptar e utilizar novas tecnologias, com atuação inovadora e empreendedora; ser capaz de reconhecer as necessidades dos usuários, formular, analisar e resolver, de forma criativa, os problemas de engenharia; adotar perspectivas multidisciplinares e transdisciplinares em sua prática; considerar os aspectos globais, políticos, econômicos, sociais, ambientais, culturais e de segurança e saúde no trabalho; atuar com isenção e comprometimento com a responsabilidade social e com o desenvolvimento sustentável (BRASIL, 2019).

Assim, a formação do engenheiro requer competências mais amplas e complexas, que meramente o conhecimento técnico, demandando, portanto, um processo de ensinoaprendizagem que possibilite esse desenvolvimento. Segundo González (2008), competências são vistas como uma combinação de conhecimentos, habilidades (intelectuais, manuais, sociais, entre outros), atitudes e valores que capacitam uma pessoa para lidar com a resolução de problemas ou a intervenção em um assunto dentro um contexto acadêmico, profissional ou social específico.

A aprendizagem ativa - que dá origem às assim denominadas "metodologias ativas" - é um termo abrangente, usado para descrever vários modelos de instrução que responsabilizam os alunos por seu próprio aprendizado, processo no qual os alunos se envolvem em "fazer as coisas e pensar sobre o que estão fazendo" na sala de aula (BONWELL; EISON, 1991). Nas metodologias ativas, o aprendizado se dá a partir de problemas e situações reais; antecipando no curso, o que os alunos vivenciarão depois na vida profissional (MORÁN, 2015). Igualmente, há uma mudança do papel exercido pelo professor, que deixa de ser uma fonte de conhecimento para ser um guia do processo de aprender, ou ainda, ao invés de um "gerente de conteúdo", se torna um facilitador da aprendizagem (HOLMES, 2019). Ou seja, estabelece-se um processo dialético onde o educador já não é o que apenas educa, mas o que, enquanto educa, é educado, em diálogo com o educando que, ao ser educado, também educa. Ambos, assim, se tornam sujeitos do processo (FREIRE, 1987).

Algumas metodologias utilizadas são a "Metodologia baseada em problemas", onde os alunos se envolvem em solução de problemas, frequentemente formulada como uma questão de pesquisa (SHWARTZ-ASHER et al., 2020); a "Metodologia baseada em projetos" que, conforme descreve Rangel (2017), consiste em produzir, por meio de etapas progressivas e contínuas, um ou mais produtos, a serem entregues em datas pré-estabelecidas. Essa metodologia promove a ligação do aluno com a parte profissional, possibilitando-o vivenciar situações reais que encontrará após a conclusão da graduação. Além dessas, outras estratégias 


\section{Evento On-line}

de aprendizagem ativa são Peer Instruction, Sala de Aula Invertida, Aprendizagem baseada em equipes, Simulações, Gamificação, etc (STROHER et al., 2018; MORAN, 2018).

Felizmente, existem muitas iniciativas da utilização das metodologias ativas em projetos pedagógicos (CASTILHO, MARIANO; ROCHA, 2019), em disciplinas ou mesmo projetos extracurriculares, a considerar os trabalhos apresentados nos últimos anos no Congresso Brasileiro de Ensino em Engenharias, segundo Vieira e Lima (2018), onde os autores realizaram uma análise bibliométrica sobre "Metodologia baseadas em Projetos". Porém, há não uma compilação sistematizada sobre o cenário atual (meados de 2020) da utilização das metodologias ativas, de forma geral, nos cursos de engenharia do Brasil. Neste contexto, o presente trabalho propôs-se a realizar uma revisão sistemática sobre o uso de metodologias ativas nos cursos de engenharia no Brasil, no período de 2011-2020.

\section{METODOLOGIA}

Este trabalho utilizou o método de pesquisa bibliográfica denominado "Revisão Sistemática de Literatura". A busca bibliográfica foi realizada a partir da plataforma Periódico Capes, nas bases Web of Science e Scopus, selecionadas por disponibilizar o formato de exportação BibTex e maior conteúdo sobre o ensino em engenharias. A pesquisa restringiu-se ao período entre 2011-2020, utilizando as seguintes strings, conforme Quadro 1.

Quadro 1: Strings utilizadas nas bases consultadas, a partir da plataforma Periódico Capes

\begin{tabular}{|c|c|}
\hline Base de Dados & String \\
\hline $\begin{array}{l}\text { Web of } \\
\text { Science }\end{array}$ & $\begin{array}{l}\text { TS }=(\text { active learning OR active methodology) AND TS=(engineering } \\
\text { education) AND CU=brazil }\end{array}$ \\
\hline Scopus & $\begin{array}{l}\text { (TITLE-ABS-KEY ("active learning" OR "active methodology" AND } \\
\text { "engineering education") AND AFFILCOUNTRY (Brazil)) AND (LIMIT- } \\
\text { TO (AFFILCOUNTRY, "Brazil")) AND (LIMIT-TO (LANGUAGE, } \\
\text { "English") OR LIMIT-TO (LANGUAGE, "Portuguese")) AND } \\
\text { (EXCLUDE (PUBYEAR, 2009) OR EXCLUDE (PUBYEAR, 2006) OR } \\
\text { EXCLUDE (PUBYEAR, 1999)) }\end{array}$ \\
\hline
\end{tabular}

Os tipos de produção pesquisados foram "Article" e "Conference paper". As listas obtidas nas bases de dados eletrônicas foram exportadas para o software Start (State of the Art Through Systematic Reviews) versão 3.0.4, elaborado pelo Laboratório de Pesquisas em Engenharia de Software, Departamento de Computação da Universidade de São Carlos (UFSCAR). O software Start4 apresenta sucessivas etapas que possibilitam a seleção e extração de informações presentes nos artigos (Figura 1).

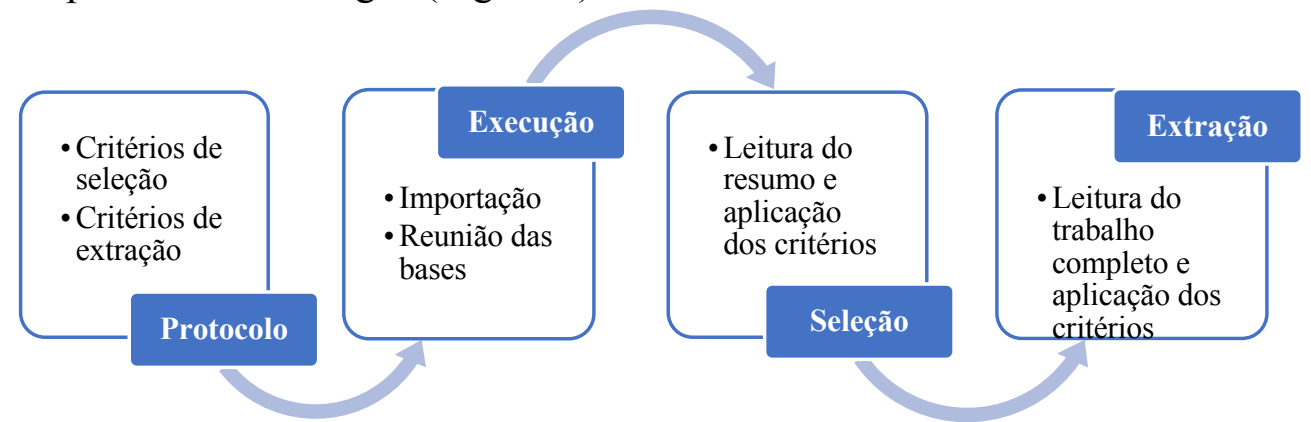

Figura 1: Fluxograma das sucessivas etapas de seleção e análise dos trabalhos, na ferramenta Start4. 
No protocolo foi delineada a pergunta-problema da pesquisa, a saber: Qual o cenário das publicações sobre o uso das metodologias ativas nos cursos de Engenharia no Brasil, no período de 2011-2020? Além disso, elaboraram-se os critérios das etapas sucessivas do processo. Para a etapa de "seleção" estabeleceram-se como critérios de inclusão (ser trabalho realizado em curso de engenharia do Brasil e ter versão completa disponível na internet) e como critérios de exclusão (não ser de curso de engenharia no Brasil e não ter trabalho completo disponível na internet). Já para a etapa de "extração" - que corresponde ao direcionamento da leitura dos trabalhos - , os critérios foram: curso [nome do curso de engenharia]; universidade [pública; privada]; região do país [Sul, Sudeste, Norte, Nordeste e Centro-oeste]; caráter da atividade [apenas na disciplina, interdisciplinar, multidisciplinar]; avaliação da percepção discente sobre a aplicação da metodologia [sim, não]; eixos [básicas, profissionalizantes, ambas]; tipo de publicação [artigo em periódico; artigo em evento científico].

\section{RESULTADOS E DISCUSSÃO}

A etapa de execução reuniu 143 trabalhos e, após a leitura do título, palavras-chave e resumo, foram excluídos os duplicados e aqueles que não se enquadraram nos critérios estabelecidos para essa etapa. Posteriormente, na etapa de extração, 113 foram lidos na íntegra, aplicando-se os critérios de inclusão e exclusão definidos anteriormente. Destes, 74 trabalhos foram incluídos e 39 excluídos (Figura 2).

A Figura 2 apresenta os resultados obtidos em cada etapa da revisão sistemática.

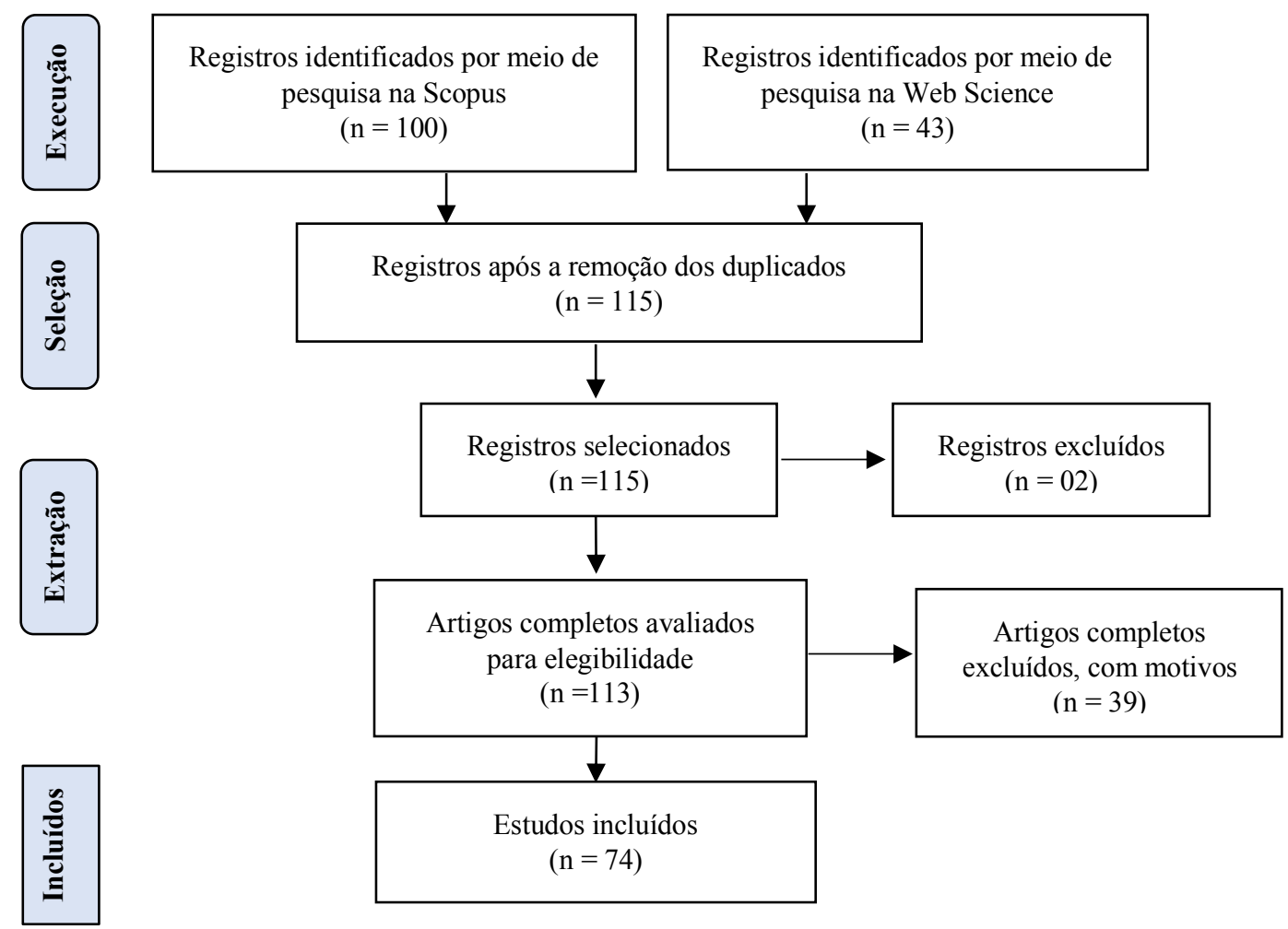

Figura 2: Fluxograma dos resultados progressivos da revisão sistemática Adaptação do modelo PRISMA (citado por GALVÃO; PANSANI; HARRAD, 2015) 
A revisão sistemática dos trabalhos evidenciou que o número de publicações sobre metodologias ativas nos cursos de engenharia no Brasil foi crescente no período analisado até 2018. Em 2011 e 2012, nenhuma publicação foi localizada; entretanto, nos anos subsequentes até 2018, houve um acréscimo significativo, porém, reduzindo em 2019 (Figura 3). Observouse que a temática esteve mais presente em publicações nos últimos cinco anos, revelando um recente interesse da comunidade acadêmica na divulgação das experiências com as metodologias ativas.

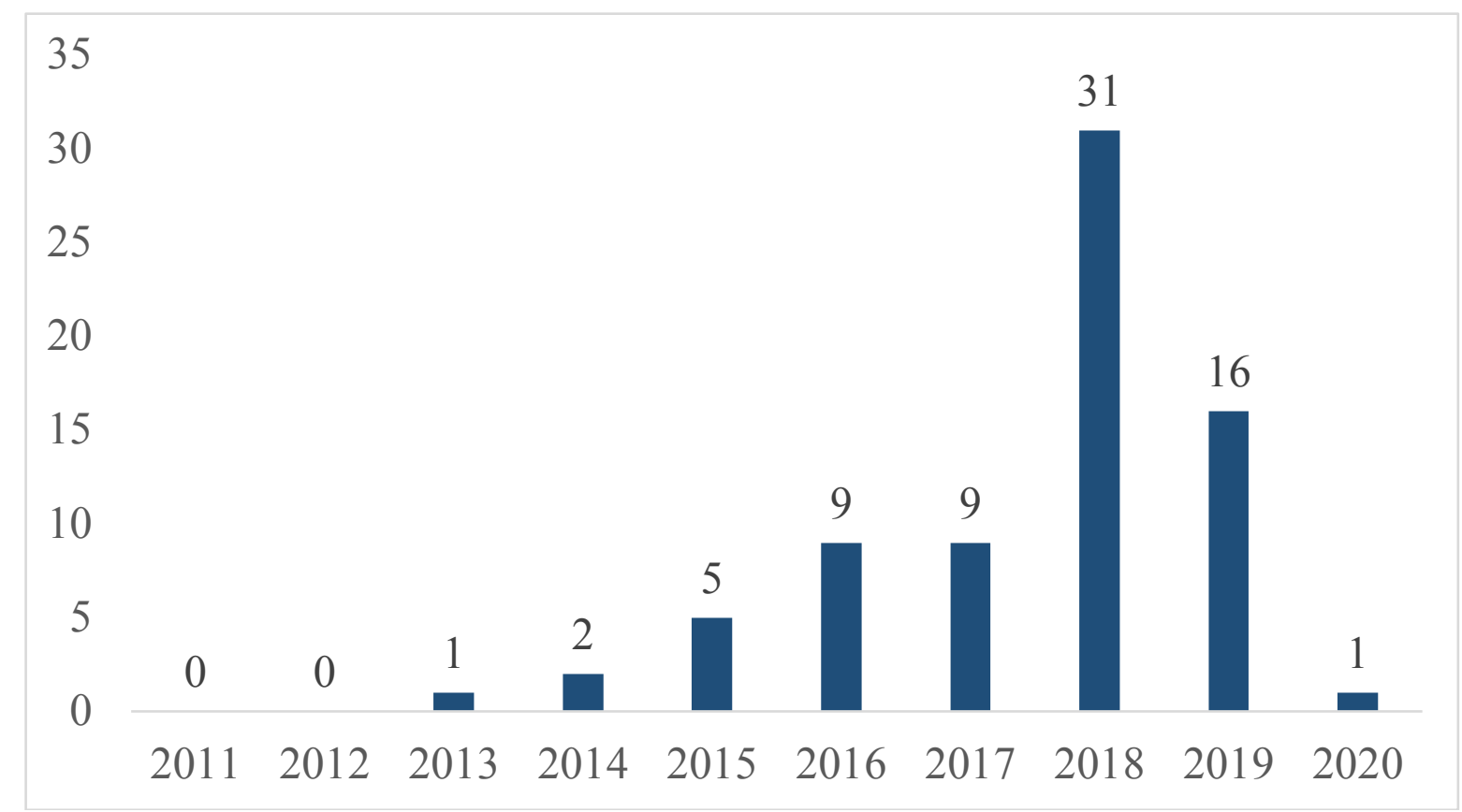

Figura 3: Número de publicação no período de 2011 a 2020.

Grande parte dos trabalhos encontrados sobre a temática de metodologias ativas foi publicada e apresentada em conferências e simpósios (85\%). O ano de 2018 foi o que apresentou o maior número de trabalhos publicados em eventos científicos, em grande parte, devido a realização em Brasília-DF, das conferências "10th International Symposium on Project Approaches in Engineering Education (PAEE)" e "15th Active Learning in Engineering Education Workshop (ALE)", onde foram apresentadas 25 publicações de autores brasileiros. Em relação aos trabalhos publicados em periódicos científicos, não foram encontradas publicações entre 2011-2014, porém nos anos seguintes, com exceção de 2016, observou-se um crescente (Quadro 2). Essa tendência também foi constatada por Pereira e Santos Júnior (2018), em revisão sistemática sobre metodologias de ensino para a formação de engenheiros no ensino superior entre 2013 e 2018. 


\section{Evento On-line}

Quadro 2: Evolução das publicações em periódicos científicos sobre metodologias ativas em engenharias no Brasil, no período de 2015-2020.

\begin{tabular}{|c|l|c|}
\hline Ano & \multicolumn{1}{|c|}{ Periódico } & N \\
\hline 2015 & -Journal of Professional Issues in Engineering Education and & 01 \\
& Practice & \\
\hline 2017 & -Revista Produção & 02 \\
& -Revista Iberoamericana de Tecnologias del Aprendizaje & 01 \\
\hline 2018 & -Computers \& Education & 01 \\
& -Educational Technology Research and Development & 01 \\
& -International Journal of Information and Communication & 01 \\
\hline 2019 & Technology Education & 01 \\
& -Interciencia & 02 \\
& -International Journal of Electrical Engineering Education & 01 \\
\hline 2020 & -European Journal of Physics & 01 \\
\hline & -International Journal of Engineering Education & 12 \\
\hline
\end{tabular}

Dos trabalhos analisados, 53\% são originários de autores ligados às instituições de ensino superior do Sudeste do país, sendo que o Nordeste, Sul e Centro-oeste apresentaram percentagens de 13, 15 e $18 \%$, respectivamente, enquanto que, para a região Norte, apenas 01\%. Ademais, $65 \%$ das instituições de ensino são públicas, devendo-se destacar a Universidade de São Paulo (USP) e a Universidade de Brasília (UnB), com maior expressão em número de publicações.

Em relação ao tipo de metodologia ativa utilizada nos trabalhos, observou-se uma predominância de Metodologia baseada em projetos e Metodologia baseada em equipes/times, seguidamente da Metodologia baseada em problemas. Metodologias como Sala de aula invertida, uso de Simulação e protótipos e Gamificação também foram utilizadas, porém em menor número de publicações. Além destas, outras metodologias também foram citadas, mas de forma pontual (Figura 4). A metodologia baseada em projetos é bastante utilizada em 


\section{Evento On-line}

diferentes cursos de engenharia no mundo, incluindo o seu uso como estratégia pedagógica nos currículos (GUERRA; ULSETH; KOLMOS, 2017).

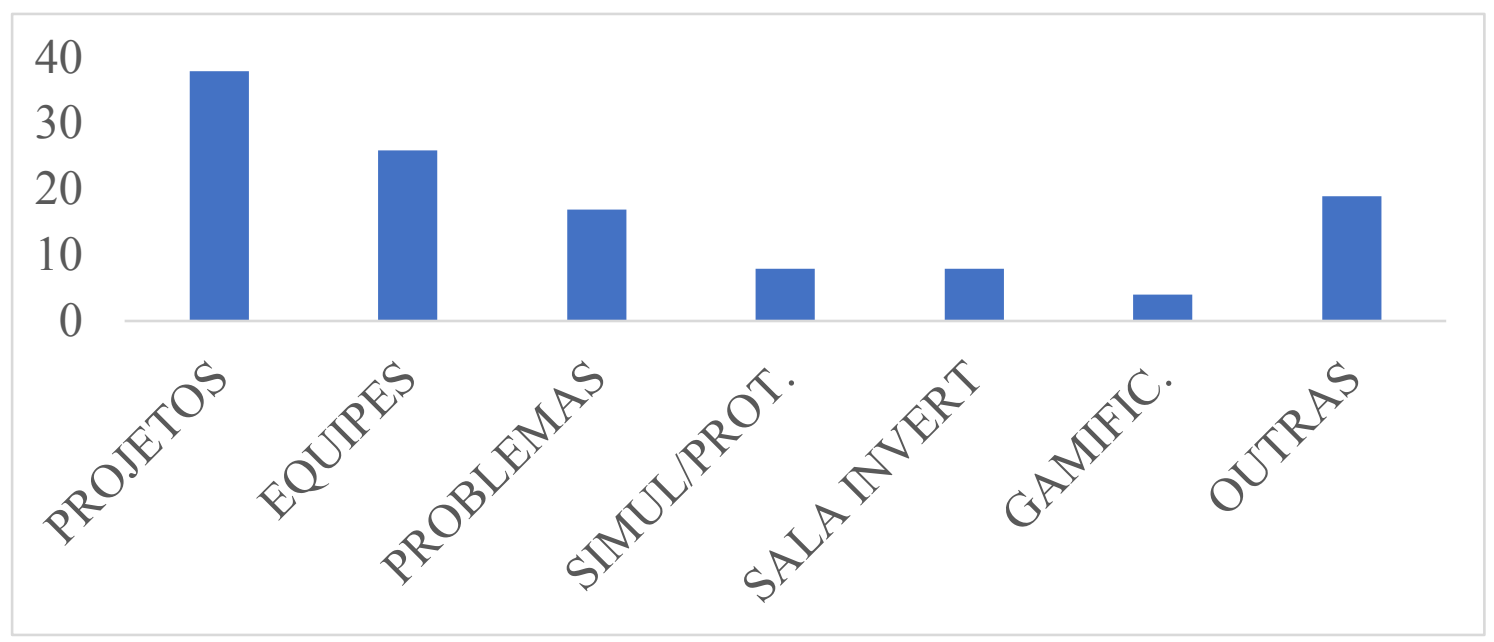

Figura 4: Número de trabalhos classificados de acordo com as metodologias ativas utilizadas, em cursos de engenharia no Brasil, no período de 2011 a 2020.

Em muitos trabalhos, observou-se o uso de mais de uma metodologia simultaneamente, geralmente associando metodologia baseada em projetos, baseada em problemas e em equipes. Segundo Kolmos (2017), o processo de aprendizagem é baseado na interação entre os alunos e seu instrutor, por um lado, e os alunos entre si, por outro. Esse ambiente possibilita que os alunos aprendam a analisar o contexto de uma perspectiva analítica crítica e aprendem os valores democráticos nas equipes por meio de discussão acadêmica, negociação, colaboração, argumentação, discordância e concordância.

Os cursos de engenharias de computação e de software são os que mais publicaram trabalhos $(23 \%)$, seguidos das engenharias elétrica e mecânica (17\%) engenharias civil, de produção civil, de produção e ambiental (16\%), engenharias química e industrial (15\%) e engenharia de automação e controle (7\%). No entanto, $16 \%$ não definiram os cursos, apenas utilizaram a denominação genérica "engenharias"; outros $6 \%$ referem-se a outras engenharias, citadas de forma pontual.

Observou-se um predomínio da utilização das metodologias ativas em disciplinas profissionalizantes (Figura 5a), de forma isolada, ou seja, apenas aplicada em uma disciplina (Figura 5b), em turmas com até 50 alunos, porém um percentual significativo não descreveu o tamanho das turmas (Figura 5c). Em todos os trabalhos analisados, os autores descrevem a 


\section{Evento On-line}

eficiência da aplicação no aprendizado dos alunos e $70 \%$ deles também realizaram uma avaliação considerando a percepção dos alunos sobre a metodologia utilizada (Figura 5d).
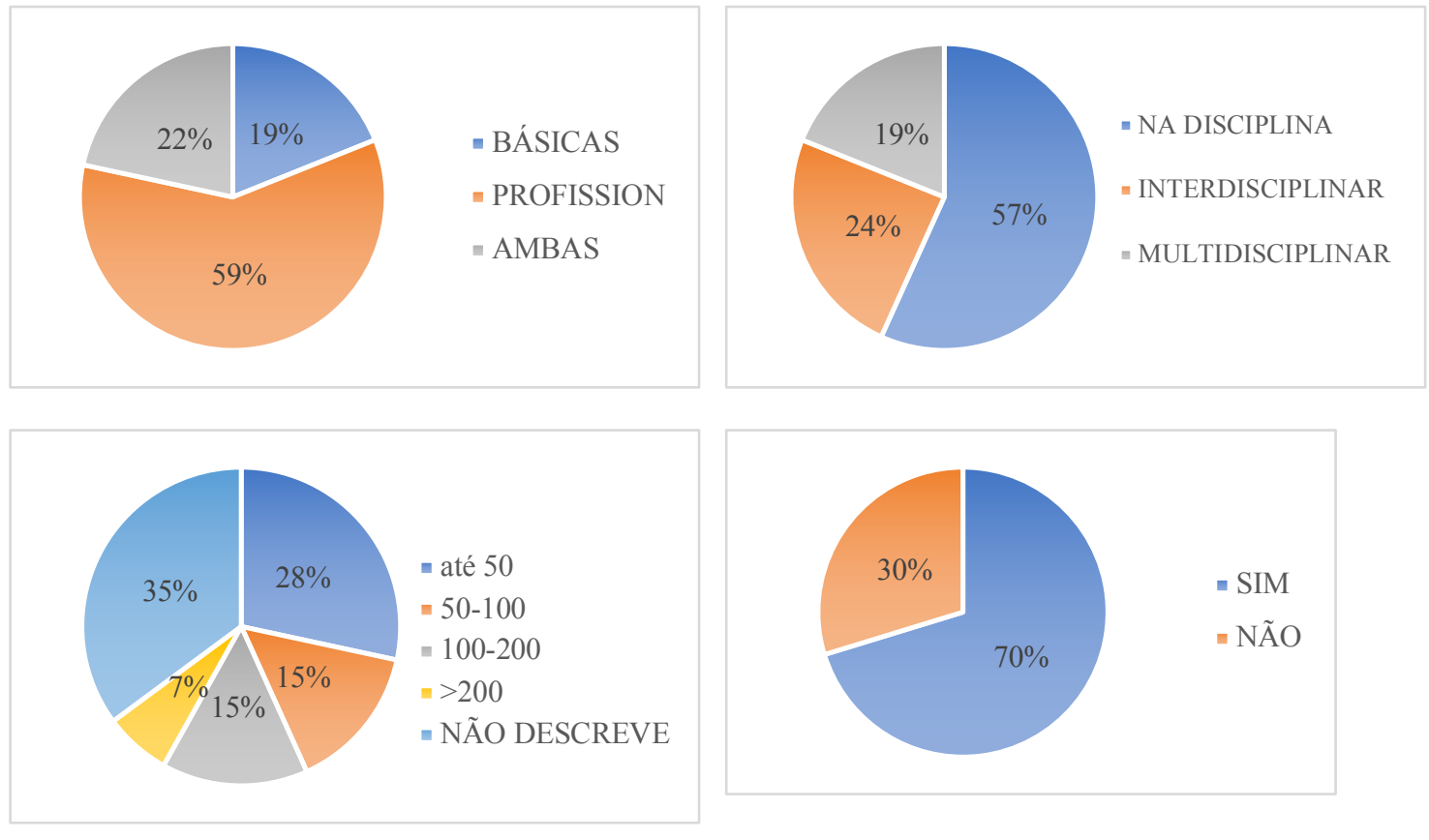

Figura 5: Dados obtidos dos critérios eixo programático, caráter da atividade, tamanho das turmas e aplicação da avaliação da metodologia ativa pelos alunos, em cursos de engenharia no Brasil, no período de 2011-2020

Sabe-se que há também um número significativo de publicações sobre metodologias ativas anualmente, no Congresso Brasileiro de Ensino em Engenharia (COBENGE), porém os anais do congresso não estão indexados nas bases pesquisadas. Uma busca pelos termos "metodologia(s) ativa(s)" ou "aprendizagem(ns) ativa(s)" nos anais desses eventos registraram 16, 36, 59 e 59 resumos expandidos publicados em 2016, 2017, 2018 e 2019, respectivamente (COBENGE, 2016, 2017, 2018, 2019), confirmando o aumento do interesse pelo tema entre os docentes brasileiros.

\section{CONSIDERAÇÕES FINAIS}

A utilização de metodologias ativas nos cursos de engenharia no Brasil assumiu maior expressão nas publicações em periódicos e eventos científicos, entre 2016-2019. A metodologia baseada em projetos é a mais utilizada. Os cursos de engenharia de computação e de software tem se destacado na divulgação dos resultados da utilização das metodologias ativas. Ademais, observou-se que todos os trabalhos analisados destacam a relevância das metodologias ativas na formação acadêmica dos futuros engenheiros, já em concordância às Diretrizes Curriculares Nacionais, promulgada em 2019.

A revisão sistemática realizada possibilitou a reflexão sobre alguns pontos que podem dificultar a implementação das metodologias ativas nos currículos de engenharia. Destaca-se o fato de que a maioria dos trabalhos foi realizada apenas em uma disciplina, o que pode limitar a condução de experiências com caráter inter e multidisciplinar. As atividades foram aplicadas em turmas reduzidas, reduzindo sua abrangência ao corpo discente do curso, que pode ser consequência da aplicação em disciplinas isoladas. Por outro lado, surpreendentemente, as 
disciplinas profissionalizantes, onde em geral, os professores não têm uma formação didáticapedagógica, apresentaram uma maior percentagem de publicações sobre metodologias ativas em relação às do ciclo básico. Essas experiências exemplificam o êxito da utilização dessas metodologias e sinalizam que há uma mudança significativa em curso na relação ensinoaprendizagem na formação profissional dos engenheiros.

\section{REFERÊNCIAS}

BONWELL, C. C.; EISON, J. A. Active Learning: creating excitement in the classroom. Washington (United States): The George Washington University, 1991.

BRASIL. Ministério da Educação. Conselho Nacional de Educação. Diretrizes Curriculares Nacionais do Curso de Graduação em Engenharia. Parecer CNE/CES No: 1/2019. D.O.U. de 23/4/2019, Seção 1, Pág. 109. Brasília (Brasil): Ministério da Educação, 2019.

CASTILHO, M. L.; MARIANO, A. M.; ROCHA, M.. Active methodologies: a proposal of practical steps for building an integrated assessment tool for national curricular guidelines. In: Anais...PAEE/ALE'2019, 11th International Symposium on Project Approaches in Engineering Education (PAEE) and 16th Active Learning in Engineering Education Workshop (ALE).

COBENGE. Inovação no ensino aprendizagem em engenharia Anais... XLV Congresso Brasileiro de Ensino em Engenharia, 2017.

COBENGE. Educação inovadora para uma engenharia sustentável. Anais... XLVI Congresso Brasileiro De Ensino Em Engenharia 2018

COBENGE. Formação por competência na engenharia no contexto da globalização 4.0. Anais... XLVII CONGRESSO BRASILEIRO DE ENSINO EM ENGENHARIA. 2019

FREIRE P. Pedagogia do oprimido. 17. Ed. Rio de janeiro: Paz e Terra; 1987.

FREIRE, P. de SÁ. Aumente a qualidade e quantidade de suas publicações científicas: manual para elaboração de projetos e artigos científicos. Curitiba- PR: Editora CRV, 2013.

GALVÃO, T. F.; PANSANI, T. de S. A.; HARRAD, D. Principais itens para relatar Revisões sistemáticas e Meta-análises: A recomendação PRISMA. Epidemiologia e Serviços de Saúde, v. 24, p. 335-342, 2015.

GONZÁLEZ, M. R. El enfoque por competencias en el EEES y sus implicaciones en la enseñanza y el aprendizaje. Tendencias Pedagógicas, v. 13, p. 79-105, 2008.

GUERRA, A.; ULSETH, R.; KOLMOS, A. (ED.). PBL in engineering education: international perspectives on curriculum change. Springer, 2017. 148p.

HOLMES, A. G. D. Constructivist learning in university undergraduate programmes. Has constructivism been fully embraced? Is there clear evidence that constructivist principles have been applied to all aspects of contemporary university undergraduate study?. Shanlax 
International Journal of Education, v. 8, n. 1, 2019KOLMOS, A. PBL curriculum strategies: From course based PBL to a systemic PBL approach. In: PBL in engineering education: international perspectives on curriculum change. Springer, 2017. p. 1-12.

PEREIRA, C. S.; DOS SANTOS JUNIOR, G.. Metodologias de ensino para a formação de engenheiros no ensino superior: uma revisão sistemática. Laplage em revista, v. 4, n. 3, p. 180 $189,2018$.

RANGEL, M. Métodos de ensino para a aprendizagem e a dinamização das aulas. 3. ed. São Paulo (Brasil): Papirus, 2007.

RODRIGUES da SILVA, A. N. A problem-project-practice based learning approach for transportation planning education. In: PBL 2010 International Conference. São Paulo, 2010.

SHWARTZ-ASHER, D. et al. Engineering teachers' assessment knowledge in active and project-based learning. In: Active Learning In College Science. Springer, Cham, 2020. P. 851869.

STROHER, J. N. et al. Estratégias pedagógicas inovadoras compreendidas como metodologias ativas. Revista Thema, v. 15, n. 2, p. 734-747, 2018.

VILLAS-BOAS, V.; MATTASOGLIO NETO, O. Aprendizagem ativa na educação em engenharia. In: Congresso brasileiro de educação em engenharia,-Cobenge, Blumenau, 2011.

\section{SYSTEMATIC REVIEW ON THE USE OF ACTIVE METHODOLOGIES IN ENGINEERING COURSES IN BRAZIL}

Abstract: The National Curriculum Guidelines for the Engineering Graduation Course spell out a clear orientation for adopting active methodologies. This present work proposed a systematic review on the use of these methodologies in Brazil's engineering courses between 2011 and 2020. The bibliographic search was carried out on the Web of Science and Scopus databases, and 143 works published in journals and scientific events were retrieved. After exporting the papers to the Start $4{ }^{\circledR}$ software, 74 were analyzed according to previously established criteria. Most of them (85\%) were published in proceedings of scientific events, and 65\% were held in public higher education institutions. 53\% are linked to institutions in the Southeast region of the country, with the Midwest, South, and Northeast having percentages of 18,15 and 13, respectively, while the North region only 01\%. There was a predominance of the methodology based on projects and teams, followed by the problems-based methodology. Computer and software engineering courses are the ones that have had the most published papers (23\%), followed by electrical and mechanical engineering (17\%) civil, civil production, production and environmental engineering (16\%), chemical and industrial (15\%). There was a preponderance of use in professionalizing disciplines, individually, in classes with up to 50 students. The vast majority of papers present an analysis of student perception about the use of the methodology. It is concluded that it is still necessary to foster inter and multidisciplinary character in the curricula, developing more complex skills and expertise, which must include all students.

Keywords: Teaching-learning. Curriculum guidelines. Active learning. State of art 